\title{
Activation of BDNF by transcription factor Nrf2 contributes to antidepressant-like actions in rodents
}

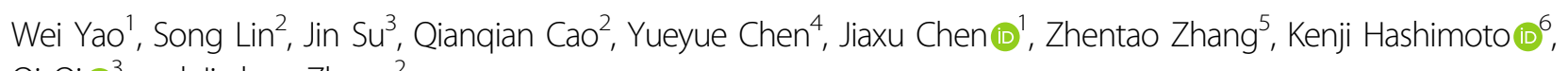
Qi Qi $\mathbb{1}^{3}$ and Ji-chun Zhang ${ }^{2}$

\begin{abstract}
The transcription factor erythroid 2-related factor 2 (Nrf2) and brain-derived neurotrophic factor (BDNF) play a key role in depression. However, the molecular mechanisms underlying the crosstalk between Nrf2 and BDNF in depression remain unclear. We examined whether Nrf2 regulates the transcription of $B d n f$ by binding to its exon I promoter. Furthermore, the role of Nrf2 and BDNF in the brain regions from mice with depression-like phenotypes was examined. Nrf2 regulated the transcription of Bdnf by binding to its exon I promoter. Activation of Nrf2 by sulforaphane (SFN) showed fast-acting antidepressant-like effects in mice by activating BDNF as well as by inhibiting the expression of its transcriptional repressors (HDAC2, mSin3A, and MeCP2) and revising abnormal synaptic transmission. In contrast, SFN did not affect the protein expression of BDNF and its transcriptional repressor proteins in the medial prefrontal cortex (MPFC) and hippocampus, nor did it reduce depression-like behaviors and abnormal synaptic transmission in Nrf2 knockout mice. In the mouse model of chronic social defeat stress (CSDS), protein levels of Nrf2 and BDNF in the MPFC and hippocampus were lower than those of control and CSDS-resilient mice. In contrast, the protein levels of BDNF transcriptional repressors in the CSDS-susceptible mice were higher than those of control and CSDS-resilient mice. These data suggest that Nrf2 activation increases the expression of Bdnf and decreases the expression of its transcriptional repressors, which result in fast-acting antidepressant-like actions. Furthermore, abnormalities in crosstalk between Nrf2 and BDNF may contribute to the resilience versus susceptibility of mice against CSDS.
\end{abstract}

\section{Introduction}

The World Health Organization estimates that major depressive disorder (MDD) is the most common psychiatric disorder worldwide, which affects more than 264 million individuals of all ages across the globe. As a result, MDD is a major contributor to the global burden of disease ${ }^{1}$. However, the precise molecular and

\footnotetext{
Correspondence: Kenji Hashimoto (hashimoto@faculty.chiba-u.jp) or Qi Qi (qiqikc@jnu.edu.cn) or Ji-chun Zhang (jczhang@jnu.edu.cn)

${ }^{1}$ Formula-Pattern Research Center, School of Traditional Chinese Medicine, Jinan University, 510632 Guangzhou, China

${ }^{2}$ Department of Physiology, School of Medicine, Jinan University, 510632

Guangzhou, China

Full list of author information is available at the end of the article

These authors contributed equally: Wei Yao, Song Lin.
}

cellular mechanisms underlying depression remain misunderstood.

Nuclear factor erythroid 2-related factor 2 (Nrf2) is a key transcription factor that regulates antioxidant and anti-inflammatory responses ${ }^{2-7}$. Accumulating evidence suggests a crucial role of Nrf2 in the pathogenesis of depression ${ }^{8,9}$. We reported that Nrf2 activator sulforaphane (SFN) showed antidepressant-like effects in the lipopolysaccharide (LPS)-induced and chronic social defeat stress (CSDS) models of depression by stimulating the expression of brain-derived neurotrophic factor $(\mathrm{BDNF})^{8,10,11}$, and that Nrf2 activators such as TBE-31 and MCE-1 showed antidepressant-like effects in LPSinduced model of depression ${ }^{12}$. Furthermore, we reported

\section{(c) The Author(s) 2021}

(c) (i) Open Access This article is licensed under a Creative Commons Attribution 4.0 International License, which permits use, sharing, adaptation, distribution and reproduction in any medium or format, as long as you give appropriate credit to the original author(s) and the source, provide a link to the Creative Commons license, and indicate if changes were made. The images or other third party material in this article are included in the article's Creative Commons license, unless indicated otherwise in a credit line to the material. If material is not included in the article's Creative Commons license and your intended use is not permitted by statutory regulation or exceeds the permitted use, you will need to obtain permission directly from the copyright holder. To view a copy of this license, visit http://creativecommons.org/licenses/by/4.0/. 
that the Nrf2 knockout (KO) mice showed decreased levels of BDNF and its receptor tropomyosin-receptorkinase $B($ TrkB) in the brain, thus resulting in depressionlike behaviors ${ }^{11}$. Furthermore, we found that the TrkB agonist 7,8-dihydroxyavone produced antidepressant-like effects in Nrf2 $\mathrm{KO}$ mice $^{11}$. A recent study demonstrated that seasonal changes in the Nrf2 pathway may regulate winter depression-like behaviors ${ }^{13}$. In the learned helplessness (LH) paradigm, the protein levels of Nrf2 and BDNF in the medial prefrontal cortex (mPFC) and hippocampus from LH (susceptible) rats were lower than those of the control and non-LH (resilient) rats ${ }^{14,15}$. Collectively, abnormalities in Nrf2 and BDNF crosstalk in the brain may play a role in causing depression-like phenotypes in rodents.

Multiple lines of evidence support the role of BDNF-TrkB signaling in the pathophysiology of depression and the therapeutic mechanisms of antidepressants and candidates including ketamine and D-serine ${ }^{16-26}$. We reported reduced levels of BDNF in the $\mathrm{MPFC}$ and hippocampus of rodents with depression-like phenotypes ${ }^{15,27-29}$. In contrast, we did not find significant changes in BDNF levels between the non-LH (resilient) rats and control rats, thus suggesting that regional differences in BDNF levels in rat brain may promote resilience to inescapable electric stress ${ }^{30}$. Moreover, the overexpression of BDNF in the hippocampus promoted resilience to stress ${ }^{31}$. Transgenic mice overexpressing the full-length $\operatorname{TrkB}\left(\operatorname{TrkB} \mathrm{TK}^{+}\right)$protein, which had an overactive TrkB-PLCY signaling, exhibited decreased depression-like actions with modified expressions of several plasticity-related genes ${ }^{32-34}$. BDNF heterozygous mice, which exhibit a $\sim 50 \%$ decrease in brain levels of BDNF compared with control mice, showed increased vulnerability to developing consequences of stress exposure $^{17}$. These results show that decreased levels of BDNF in the MPFC and hippocampus may contribute to the pathophysiology of depression, whereas activation of the BDNF-TrkB signaling pathway may promote resilience. Taken together, it is important to study the relationship between the Nrf2 and BDNF in rodents with depressionlike behaviors. However, the precise molecular mechanisms underlying Nrf2 and BDNF crosstalk in depression are currently unknown.

In the present study, we hypothesized that Nrf2 may regulate the transcription of $B d n f$ by binding to its promoter and causing the dissociation of transcriptional repressors (i.e., $\mathrm{HDAC} 2, \mathrm{mSin} 3 \mathrm{a}$, and $\mathrm{MeCP} 2$ ) from the promoter. First, we examined whether Nrf2 activation can affect the expression of BDNF and BDNF transcriptional repressor (i.e., HDAC2, mSin3a, and MeCP2). Second, we examined whether Nrf2 regulates $B d n f$ transcription using luciferase assay and chromatin immunoprecipitation (ChIP) assay. Third, we examined the role of Nrf2 and
BDNF crosstalk on the depression-like phenotypes in LPS-treated mice and Nrf2 KO mice. Finally, we examined the role of Nrf2 and BDNF in resilience versus susceptibility in mice after CSDS.

\section{Materials and methods}

Mice, cell lines, primer information, antibody information, and drug treatment

Male adult C57BL/6 mice (8 weeks old, 20-25 g each, Guangdong Experimental Animal Center), CD1 mice (14 weeks old, 40-45 g each, Guangdong Experimental Animal Center), and male adult Nrf2 homozygous $\mathrm{KO}$ mice $\left(\mathrm{Nrf2}^{-/-}\right)$were used in experiments. Age-matched animals from each genotype were randomly allocated to experimental groups. The sample sizes were based on previous experience with the experimental design ${ }^{10}$. Since several batches of mice were tested independently and pooled together for final analyses. Therefore, the group sizes are not exactly the same. The criteria were not preestablished. The animals were housed under controlled temperature and kept on a 12-h light/dark cycle (lights on between 07:00 and 19:00), with ad libitum access to food and water. The protocol was approved by the Jinan University Institutional Animal Care and Use Committee. All experiments were carried out following the Guide for Animal Experimentation of Jinan University. HEK293 or Neuro-2a cells were a gift from Dr. Zhentao Zhang (Department of Neurology, Renmin Hospital of Wuhan University, Wuhan, China) and cultured in high-glucose DMEM supplemented with $10 \%$ fetal bovine serum (Excell Bio.) and penicillin (100 units $/ \mathrm{mL})$-streptomycin $(100 \mu \mathrm{g} / \mathrm{mL})$ (all from Hyclone). Cells were incubated at $37^{\circ} \mathrm{C}$ in a humidified incubator containing $5 \% \mathrm{CO}_{2}$. All the cell lines were tested for mycoplasma contamination.

Primer sequences used were for Nrf2, Bdnf, and Gapdh quantitative real-time PCR assay as follows: forward $5^{\prime}$ CGAGATATACGCAGGAGAGGTAAGA $3^{\prime}$; reverse $5^{\prime}$ GCTCGACAATGTTCTCCAGCTT $3^{\prime}$ for $N r f 2$, forward 5' TTGTTTTGTGCCGTTTACCA3'; reverse 5' GGTAA GAGAGCCAGCCACTG $3^{\prime}$ for $B d n f$, and forward, $5^{\prime}$ ATGACATCAAGAAGGTGGTG 3', reverse, 5' CATA CCAGGAAATGAGCTTG 3' for Gapdh.

The following antibodies were purchased from Abcam: Nrf2 (ab137550), BDNF (ab108319), HDAC2 (ab12169), mSin3A (ab3479), and MeCP2 (ab2828). The beta-actin antibody was purchased from EarthOx.

On the day of injection, fresh solutions were prepared by dissolving drug compounds in sterile endotoxin-free isotonic saline. LPS $(0.5 \mathrm{mg} / \mathrm{kg}$; L-4130, serotype 0111 : B4, Sigma-Aldrich, St. Louis, MO, USA) was dissolved in physiological saline. SFN (10 mg/kg; MedChemExpress, Shanghai, China) was dissolved in distilled water containing $10 \%$ corn oil. LPS $(0.5 \mathrm{mg} / \mathrm{kg})$ and SFN $(10 \mathrm{mg} /$ $\mathrm{kg}$ ) were administered intraperitoneally (i.p.) according 
to previous reports by a researcher blind to the treatment $^{10,27,28}$. $\mathrm{D}(-)$-2-amino-5-phosphonovaleric acid (AP5), 6-cyano-7-nitroquinoxaline-2,3-dione (CNQX), and Bicuculine methiodide (BMI) were purchased from Tocris Bioscience. AP5 $(100 \mu \mathrm{M})$ and CNQX $(20 \mu \mathrm{M})$ were used to verify spontaneous excitatory postsynaptic currents (sEPSCs) and BMI $(20 \mu \mathrm{M})$ was used to verify spontaneous inhibitory postsynaptic currents (sIPSCs)

\section{CSDS model}

CSDS was induced according to previously reported procedures (for details, see Supplementary Information $)^{35-38}$.

\section{Behavioral tests}

Behavioral tests including locomotion, tail suspension test (TST), forced swimming test (FST), and 1\% sucrose preference test were performed according to previously reported procedures (for details, see Supplementary Information) ${ }^{10,27,28}$.

\section{Cell transfection}

The mouse plasmid (pcDNA3.1-Nrf2) was a gift from Dr. Masayuki Yamamoto (Tohoku University Graduate School of Medicine, Sendai, Japan). The mouse Bdnf exons I, II, and IV luciferase plasmids were structured by TsingKe Biological Technology (Wuhan, China). siRNAs were purchased from Santa Cruz. HEK293 or Neuro-2a cells were transfected using Lipofectamine 3000 (Invitrogen) according to the manufacturer's instructions for cell $48 \mathrm{~h}$.

\section{Western blotting assay, luciferase assay, ChIP assay, immunofluorescence staining, and electrophysiological recordings}

We performed western blot, luciferase assay, ChIP assay, and immunofluorescence staining and obtained electrophysiological recordings for in vitro and/or in vivo experiments (for details, see Supplementary Information).

\section{Statistical analysis}

Data are expressed as the mean \pm standard error of the mean (SEM). Data were analyzed using PASW Statistics 20 software. Data were analyzed using Student's $t$-test or one-way analysis of variance, followed by post hoc Fisher's least significant difference test. All $p$-values $<0.05$ were considered statistically significant.

\section{Results}

Role of Nrf2 in the expression of BDNF transcriptional repressors (MeCP2, HDAC2, and $\mathrm{mSin} 3 \mathrm{a}$ )

We previously reported that the Nrf2 activator SFN ameliorated the decreased expression of BDNF protein in the brain of mice with a depression-like phenotype ${ }^{10,11,27}$.
Hence, we examined to explore whether Nrf2-mediated increases in BDNF affect the expression of Bdnf transcriptional repressors (Fig. 1A-H). SFN and/or siRNANrf2 plasmids for Neuro-2a cells were used. SFN increased the mRNA and protein expression of BDNF and decreased the protein levels of HDAC2, mSin3a, and MeCP2. However, these effects by SFN were blocked by siRNA-Nrf2. Moreover, overexpression of Nrf2 in Neuro2a cells increased the mRNA and protein levels of BDNF while decreasing the protein levels of HDAC2, mSin3a, and MeCP2 (Fig. 1A-H). These results suggest that Nrf2 activation can increase BDNF mRNA and protein expression, whereas Nrf2 activation decreases the expression of HDAC2, mSin3a, and MeCP2 protein.

\section{Nrf2 mediates Bdnf transcription}

To further determine the mechanism underlying the transcriptional regulation of Nrf2 on $B d n f$, we analyzed the DNA sequences of the promoter regions in mouse $B d n f$ exons I, II, and IV promoters. We found two potential Nrf2 consensus binding motifs in $B d n f$ exon I promoter based on Nrf2 binding characteristics ${ }^{39}$ (Supplementary Fig. 1). Accordingly, we prepared a series of luciferase-conjugated constructs with the promoters for the $B d n f$ exons I, II, and IV sequences. The luciferase assay using lysates from HEK293 cells treated with SFN or co-transfected with Nrf2 or siRNA-Nrf2 and the promoter constructs showed that $B d n f$ exon I promoter might be the major binding site for Nrf2 (Fig. 2A, B). The mutation in this motif completely abolished promoter activity (Fig. 2C, D). To further confirm that Nrf2 acts as a transcription factor for $B d n f$, we carried out a ChIP assay with cells treated with SFN or Nrf2 by using an Nrf2-specific antibody. PCR analysis with genomic DNA immunoprecipitated using the Nrf2 antibody demonstrated that Nrf2 interacted with the $B d n f$ exon I promoter (Fig. 2E). Immunofluorescence staining revealed that SFN treatment caused the redistribution of $\mathrm{Nrf} 2$ and $\mathrm{MeCP} 2$ for the nucleus. We found that more Nrf2 within the nucleus and the more diffused nuclear staining pattern of MeCP2 in vehicle-treated cells became more punctate upon SFN treatment (Fig. 2F). These results suggest that Nrf2 functions as a transcription factor for $B d n f$.

\section{The roles of Nrf2 in depression-like behaviors and the expression of BDNF after LPS administration}

We examined whether Nrf2 plays a role in LPS-induced depression-like behaviors by elevating BDNF expression and inhibiting BDNF transcriptional repressor expression. First, we found that LPS $(0.5 \mathrm{mg} / \mathrm{kg}$, i.p.) significantly decreased the levels of Nrf2 and BDNF and increased the levels of $\mathrm{HDAC} 2, \mathrm{mSin} 3 \mathrm{a}$, and $\mathrm{MeCP} 2$ protein in the $\mathrm{mPFC}$ and hippocampus (Fig. 3A, B). SFN (10 mg/kg, i.p.) significantly attenuated the reduction in Nrf2 and BDNF 


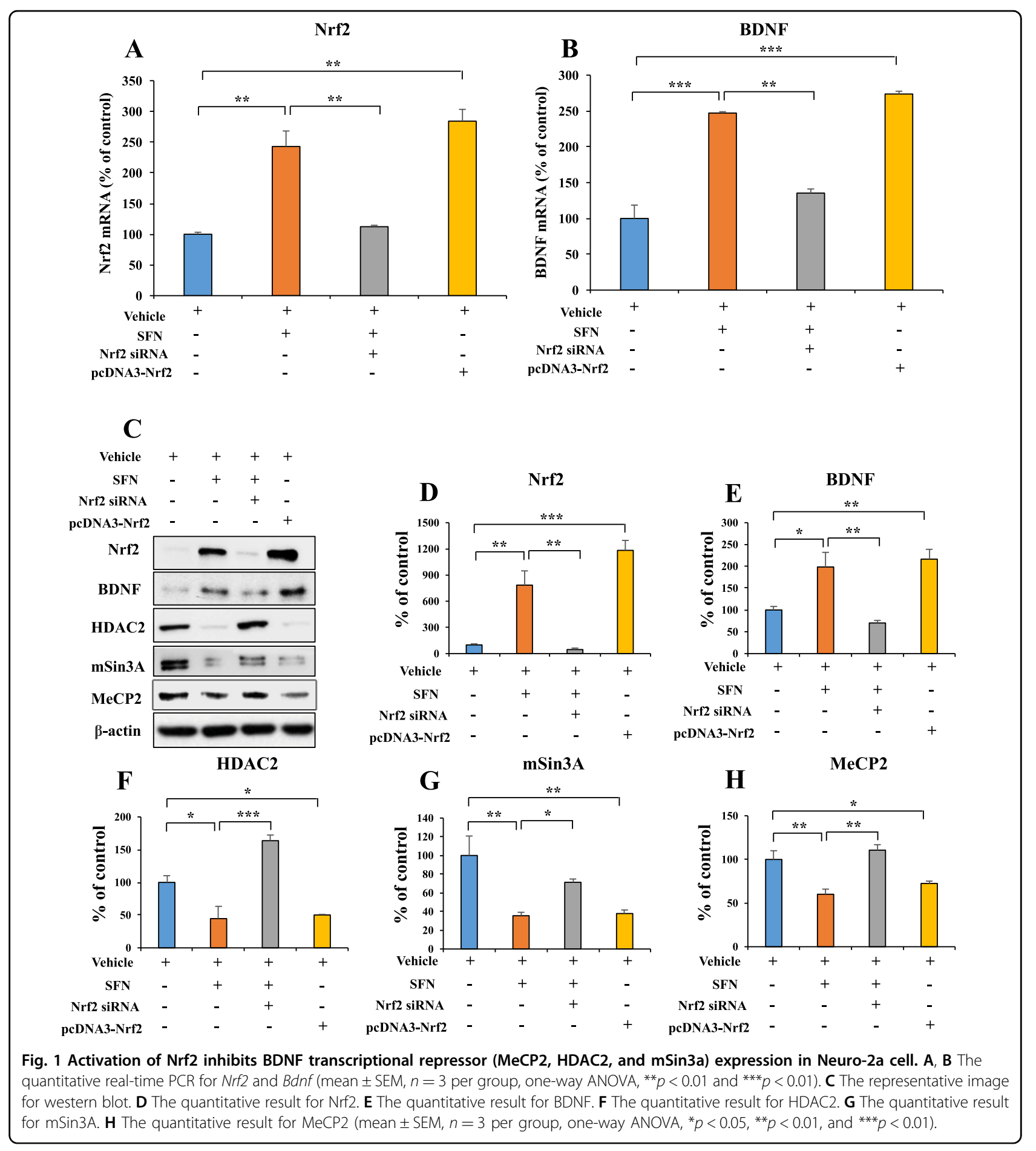

protein expression observed after LPS administration and increased the levels of MeCP2, HDAC2, and mSin3a protein in the mPFC and hippocampus (Fig. 3A, B). Next, we used immunofluorescence to visualize Nrf2 and $\mathrm{MeCP} 2$ in the selected brain regions. LPS significantly decreased the Nrf2 fluorescence intensity, whereas LPS significantly increased the MeCP2 fluorescence intensity in the mPFC and dentate gyrus (DG) of the hippocampus. SFN significantly attenuated the reduction in Nrf2 fluorescence intensity and increased the $\mathrm{MeCP} 2$ fluorescence intensity in the mPFC and DG of the hippocampus after a single dose of LPS (Supplementary Fig. 2A, B).

Moreover, we performed the behavioral tests to examine the antidepressant-like effects of SFN in LPS-induced 

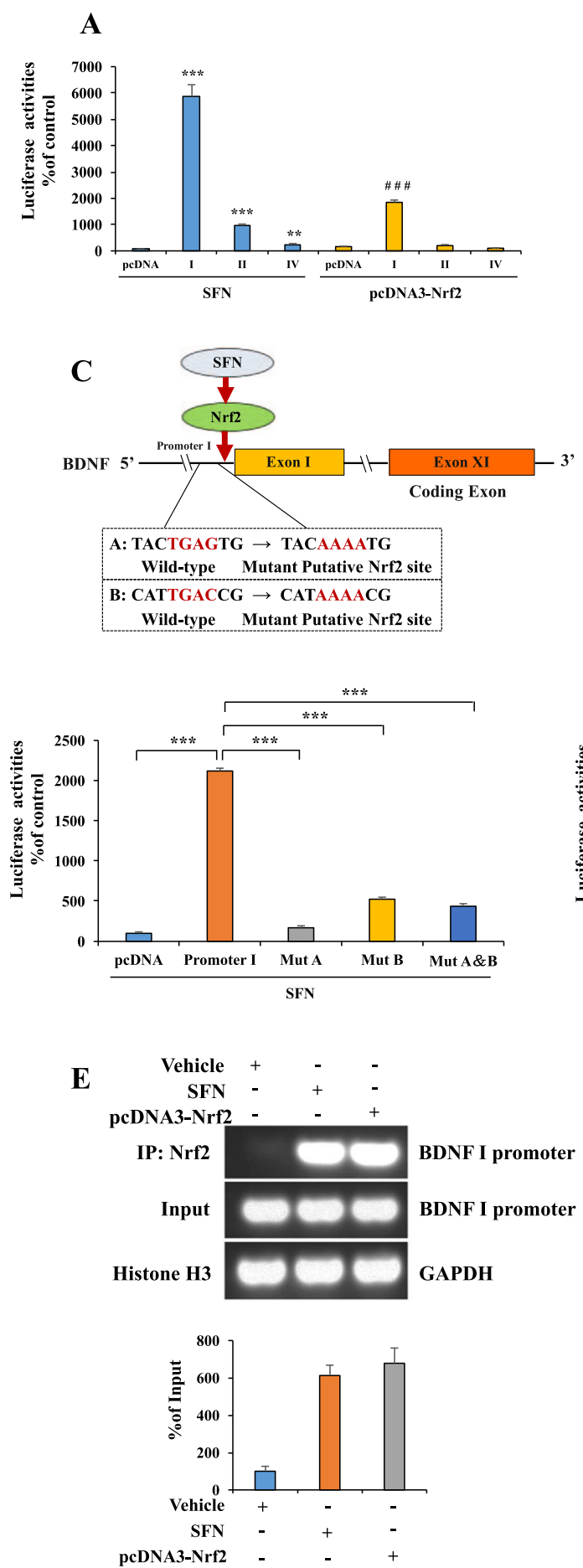

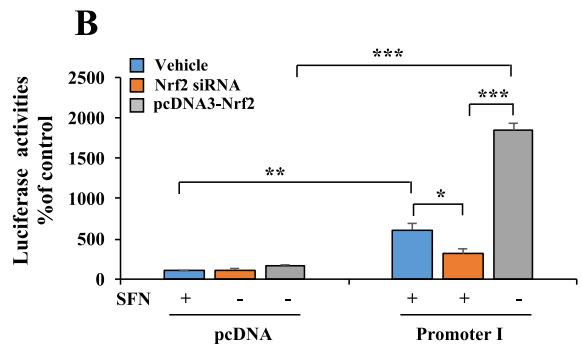

D
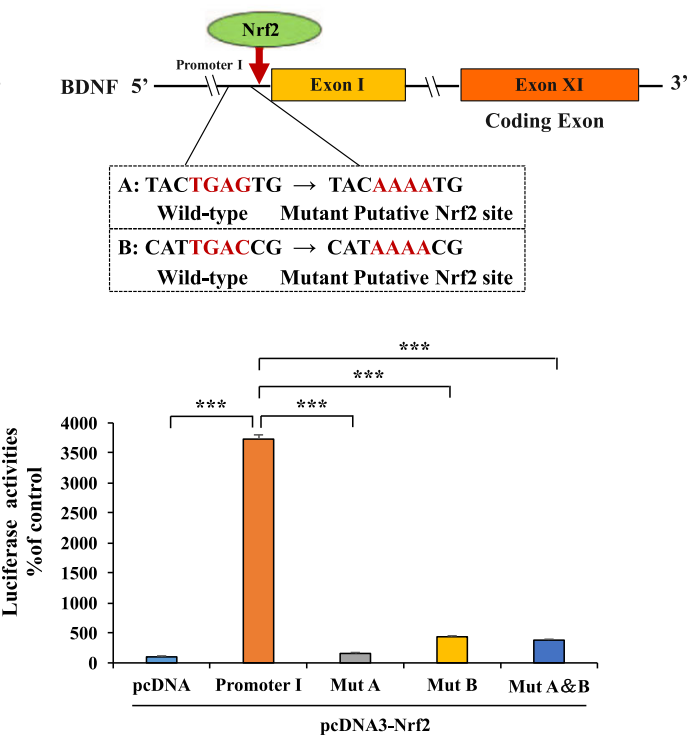

F
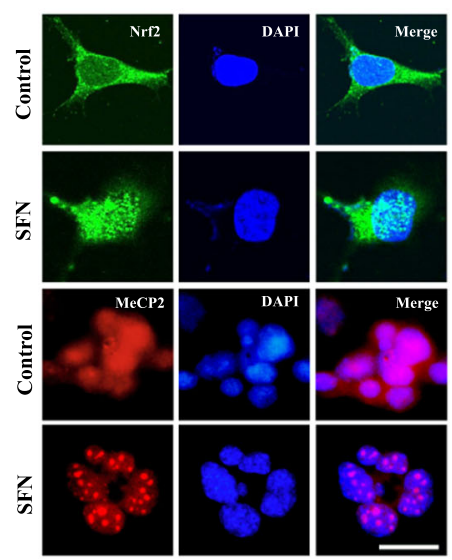

Fig. 2 Nrf2 acts as transcription factor for BDNF. A-E Nrf2 acted as a transcription factor for BDNF promoters. A, B BDNF exons I, II, and IV luciferase promoters and SFN or Nrf2 or siRNA-Nrf2 plasmids were treated into HEK293 cells (mean \pm SEM, $n=4$ per group, one-way ANOVA, * $p<$ $0.05,{ }^{* *} p<0.01,{ }^{* *} p<0.001$, and \# \# $p<0.001$ ). C, D Results obtained using the luciferase plasmids containing mutation (Mut) at the Nrf2 binding motif of BDNF exon I promoter was compared with the wild type of promoters, respectively ( $m e a n \pm S E M, n=4$ per group, one-way ANOVA, ${ }^{*} p<$ $0.05,{ }^{* *} p<0.01$, and $\left.{ }^{* * *} p<0.001\right)$. E ChIP-PCR assays demonstrated Nrf2 specifically bound to genomic DNA of BDNF exon I promoter binding motifs. The Nrf2 protein-DNA crosslinking samples were obtained from the HEK293 cells treated with SFN or Nrf2 plasmid or not (control) via coimmunoprecipitating with anti-Nrf2 antibodies. PCR was carried out by using primer pairs at BDNF exon I promoter. PCR assay also included each input sample. The positive control was demonstrated with anti-Histone $\mathrm{H} 3$ antibody coupling with GAPDH primers. F The immunofluorescence for Nrf2 and MeCP2. The SFN treated for Neuro-2a cell $24 \mathrm{~h}$. The immunofluorescence was performed for Nrf2 and MeCP2. Scale bar 50 um. 

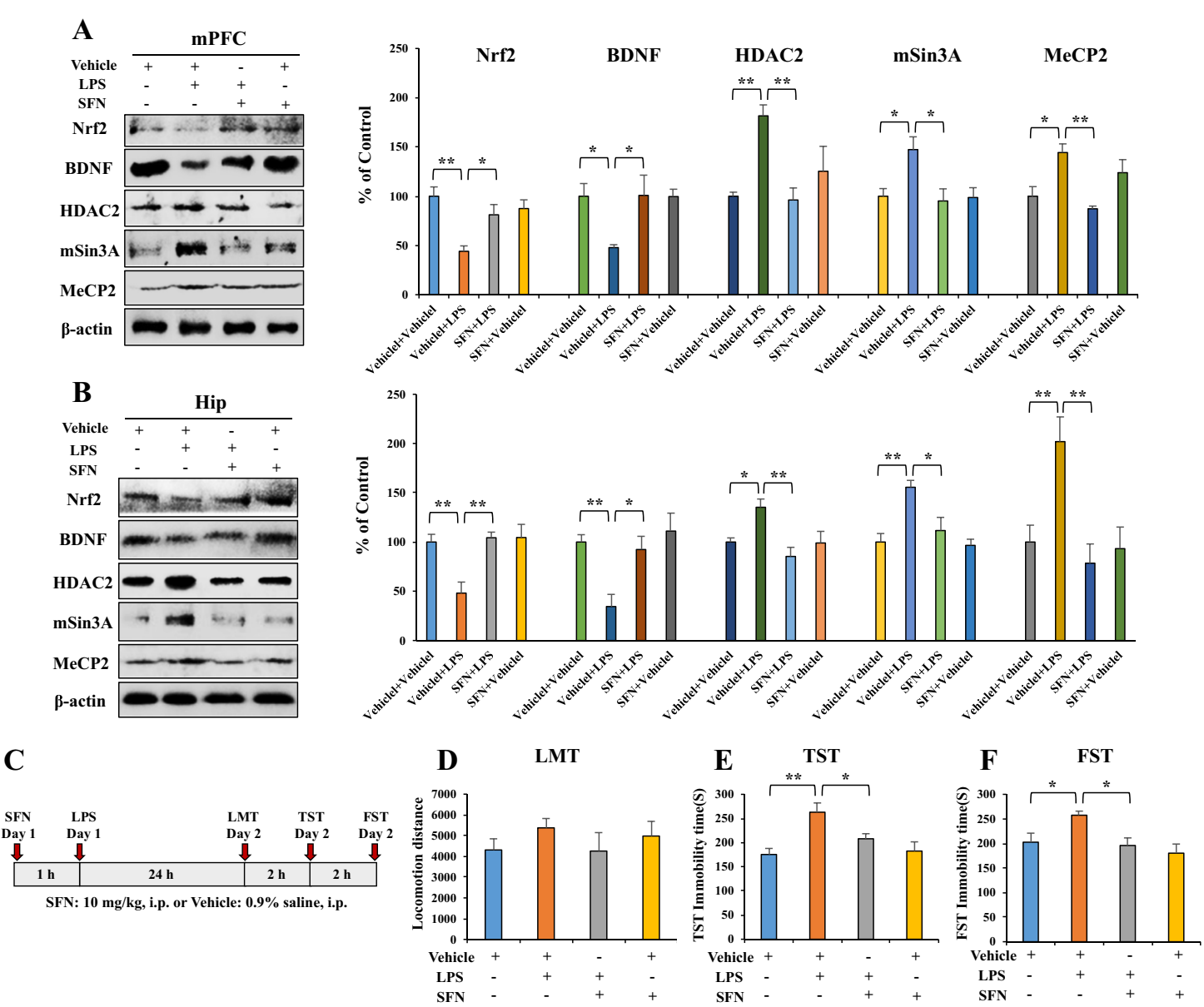

\section{G}

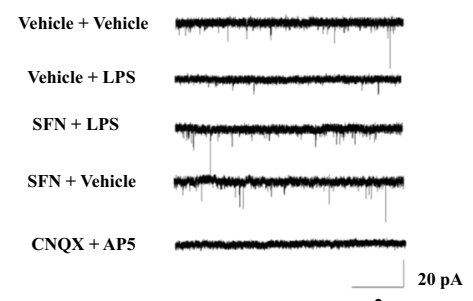

$2 \mathrm{~s}$

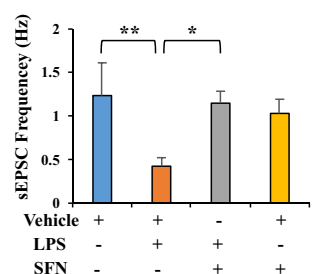

$\mathbf{H}$

mPFC sEPSC

Vehicle + Vehicle

Vehicle + LPS

SFN + Vehicle

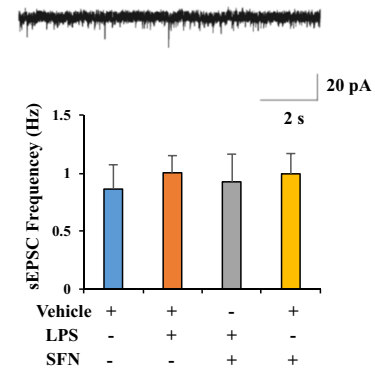

I

mPFC sIPSC

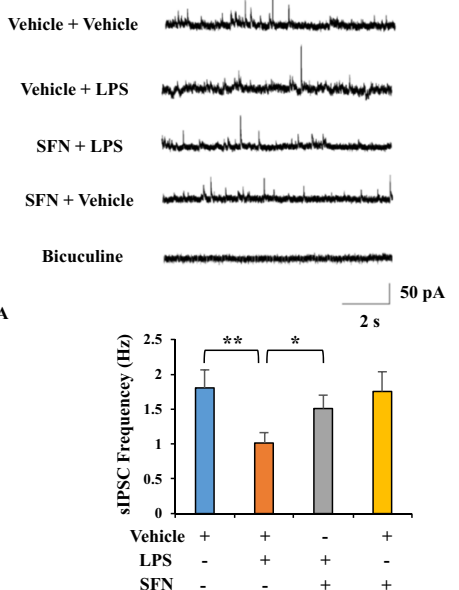

Fig. 3 SFN shows beneficial effects for depression-like behavior by altering Nrf2, BDNF, HDAC2, mSin3a, MeCP2 expression, and attenuating the decrease of frequency of sEPSC and sIPSC in hippocampus and mPFC neurons. A, B Western blot analysis for Nrf2, BDNF, and MeCP2 in MPFC and hippocampus (mean \pm SEM, $n=4$ per group, one-way ANOVA, ${ }^{*} p<0.05$ and ${ }^{* *} p<0.01$ ). $C$ The schedule of behavior test and treatment. D LMT: locomotion test, E TST: tail-suspension test, F FST: forced swimming test (mean \pm SEM, $n=7$ or 8 per group, one-way ANOVA, ${ }^{*} p<$ $\left.0.05,{ }^{* *} p<0.01\right)$. G Up: representative traces of sEPSC in hippocampal DG neurons. Scale bars represent $2 \mathrm{~s}, 20 \mathrm{pA}$. Down: histograms of sEPSC frequency in hippocampal DG neurons (mean \pm SEM,$n=7-9$ neurons of three mice per group, one-way ANOVA, $\left.{ }^{*} p<0.05,{ }^{* *} p<0.01\right)$. H Up: representative traces of sEPSC in $\mathrm{mPFC}$ neurons. Scale bars represent $2 \mathrm{~s}, 20 \mathrm{pA}$. Down: histograms of sEPSC frequency in mPFC neurons (mean \pm SEM, $n=8-10$ neurons of three mice per group). I Up: representative traces of sIPSC in mPFC neurons. Scale bars represent $2 \mathrm{~s}, 50 \mathrm{pA}$. Down: histograms of sIPSC frequency in MPFC neurons (mean \pm SEM,$n=8-10$ neurons of three mice per group, one-way ANOVA, ${ }^{*} p<0.05,{ }^{* *} p<0.01$ ). 
depression-like behaviors (Fig. 3C). There were no changes in locomotion observed among the four groups (Fig. 3D). SFN significantly attenuated the increased immobility time of TST and FST observed after LPS administration (Fig. 3E, F). However, SFN alone did not affect the immobility time during the TST and FST in control mice (Fig. 3E, F).

Previous studies showed that depression-like behaviors are associated with impaired synaptic transmission in the hippocampus and $\mathrm{mPFC}^{40-42}$, and BDNF is an important regulator of synaptic transmission ${ }^{43}$. Therefore, we obtained whole-cell patch-clamp recordings in acute hippocampus slices and recorded sEPSCs. LPS treatment significantly decreased the frequency of sEPSC without affecting the amplitude in neurons of the hippocampal DG (Fig. 3G and Supplementary Fig. 3A). Furthermore, SFN significantly attenuated the decrease in the sEPSC frequency in hippocampal DG neurons after LPS administration (Fig. 3G). However, SFN alone did not affect the frequency or amplitude of sEPSCs in hippocampal DG neurons (Supplementary Fig. 3A). sEPSC could be completely blocked by the AMPA and NMDA receptor antagonists AP5 and CNQX. We recorded sEPSCs of mPFC neurons to further determine whether LPS administration can affect glutamatergic synaptic transmission in mPFC neurons. However, LPS and SFN administration did not affect the frequency and amplitude of sEPSCs in mPFC neurons (Fig. $3 \mathrm{H}$ and Supplementary Fig. 3B). Because disrupted GABA neurotransmission in the $\mathrm{mPFC}$ contributes to the neurobiology of depression $^{44}$, we tested whether GABAergic transmission was altered after LPS injection. Surprisingly, we found that LPS treatment significantly decreased the frequency of sIPSCs without affecting the amplitude of MPFC neurons. Furthermore, this change could be reversed by SFN treatment (Fig. 3I and Supplementary Fig. 3C). SFN alone did not affect the frequency or amplitude of sIPSCs in MPFC neurons (Fig. 3I and Supplementary Fig. 3C). sIPSCs were inhibited by bicuculline methiodide (BMI), a $\mathrm{GABA}_{\mathrm{A}}$ receptor antagonist.

\section{Role of Nrf2 in depression-like phenotypes and changes in} BDNF, HDAC2, mSin3a, and MeCP2 expression

We used Nrf2 $\mathrm{KO}$ mice with a depression-like phenotype to confirm the role of Nrf2 and BDNF crosstalk in the pathogenesis of depression ${ }^{11}$. Using western blot, we analyzed BDNF, HDAC2, mSin3A, and MeCP2 protein expression in the selected brain regions from wild type (WT) mice and Nrf2 KO mice. Protein levels of BDNF in the mPFC and hippocampus of $N r f 2 \mathrm{KO}$ mice were significantly lower than those of WT mice. Furthermore, protein levels of $\mathrm{HDAC} 2, \mathrm{mSin} 3 \mathrm{~A}$, and $\mathrm{MeCP} 2$ in the $\mathrm{mPFC}$ and hippocampus were significantly higher than those of WT mice; the change in HDAC2 expression in the $\mathrm{mPFC}$ was not statistically significant (Fig. 4A, B).
Treatment of SFN did not change the expression of these proteins in Nrf2 $\mathrm{KO}$ mice (Fig. 4A, B). These data suggest the role of Nrf2 and BDNF crosstalk in the depression-like phenotypes of $N r f 2 \mathrm{KO}$ mice.

Nrf2 KO mice showed depression-like behaviors, which was consistent with our previous report ${ }^{11}$. In contrast, SFN $(10 \mathrm{mg} / \mathrm{kg})$ did not show antidepressant-like effects in $\mathrm{Nrf2}$ $\mathrm{KO}$ mice (Fig. 4C-G). There were no differences in locomotion among the three groups (Fig. 4D). Regarding the TST and FST, the increased immobility times of Nrf2 KO mice were not improved after SFN $(10 \mathrm{mg} / \mathrm{kg})$ administration (Fig. 4E, F). Furthermore, the decreased sucrose preference of Nrf2 KO mice was not improved after the administration of SFN (Fig. 4G). These data suggest the role of Nrf2 in the antidepressant-like effects of SFN.

Moreover, we also performed whole-cell patch-clamp recordings in acute hippocampus and $\mathrm{mPFC}$ slices and recorded sEPSCs and sIPSCs for Nrf2 $\mathrm{KO}$ mice. In $N r f 2 \mathrm{KO}$ mice, the frequency of sEPSC is significantly decreased without affecting the amplitude in neurons of the hippocampal DG (Fig. $4 \mathrm{H}$ and Supplementary Fig. $4 A)$. SFN $(10 \mathrm{mg} / \mathrm{kg})$ did not alter the frequency of sEPSC in Nrf2 $\mathrm{KO}$ mice (Fig. 4H).

LPS significantly decreased the frequency of sIPSC in neurons of $\mathrm{mPFC}$, and SFN $(10 \mathrm{mg} / \mathrm{kg})$ significantly attenuated the decreased frequency of sIPSC in neurons of mPFC (Fig. 3I). Therefore, we examined the frequency and amplitude of sIPSC in neurons of mPFC for $N r f 2 \mathrm{KO}$ mice. In Nrf2 KO mice, the frequency of sIPSC is significantly decreased without affecting the amplitude in neurons of the mPFC (Fig. 4I and Supplementary Fig. 4B). SFN (10 mg/kg) did not alter the frequency and amplitude of sIPSC in Nrf2 KO mice (Fig. 4I and Supplementary Fig. 4B).

\section{Role of Nrf2, BDNF, and BDNF transcriptional repressor proteins in the CSDS model}

Previously, we reported that the protein expression of Nrf2 and BDNF in the mPFC and hippocampus of LH (susceptible) rats was lower than that of control and non-LH (resilient) rats ${ }^{14,15}$. Here we used the CSDS model of depression. Using the social interaction test, we divided the CSDS-susceptible and CSDS-resilient mice (Fig. 5A-E). We observed decreased expression of Nrf2 and BDNF in the mPFC and hippocampus from CSDS-susceptible mice, but not CSDS-resilient mice, compared with that from the control (no CSDS) mice (Fig. 5E, F). In contrast, we observed increased expression of HDAC2, mSin3A, and MeCP2 in the MPFC and hippocampus from CSDS-susceptible mice, but not CSDS-resilient mice, compared with that from the control (no CSDS) mice (Fig. 5E, F). There were no differences in change of these transcriptional repressor proteins between CSDS-resilient mice and control (no CSDS) mice (Fig. 5E, F). 


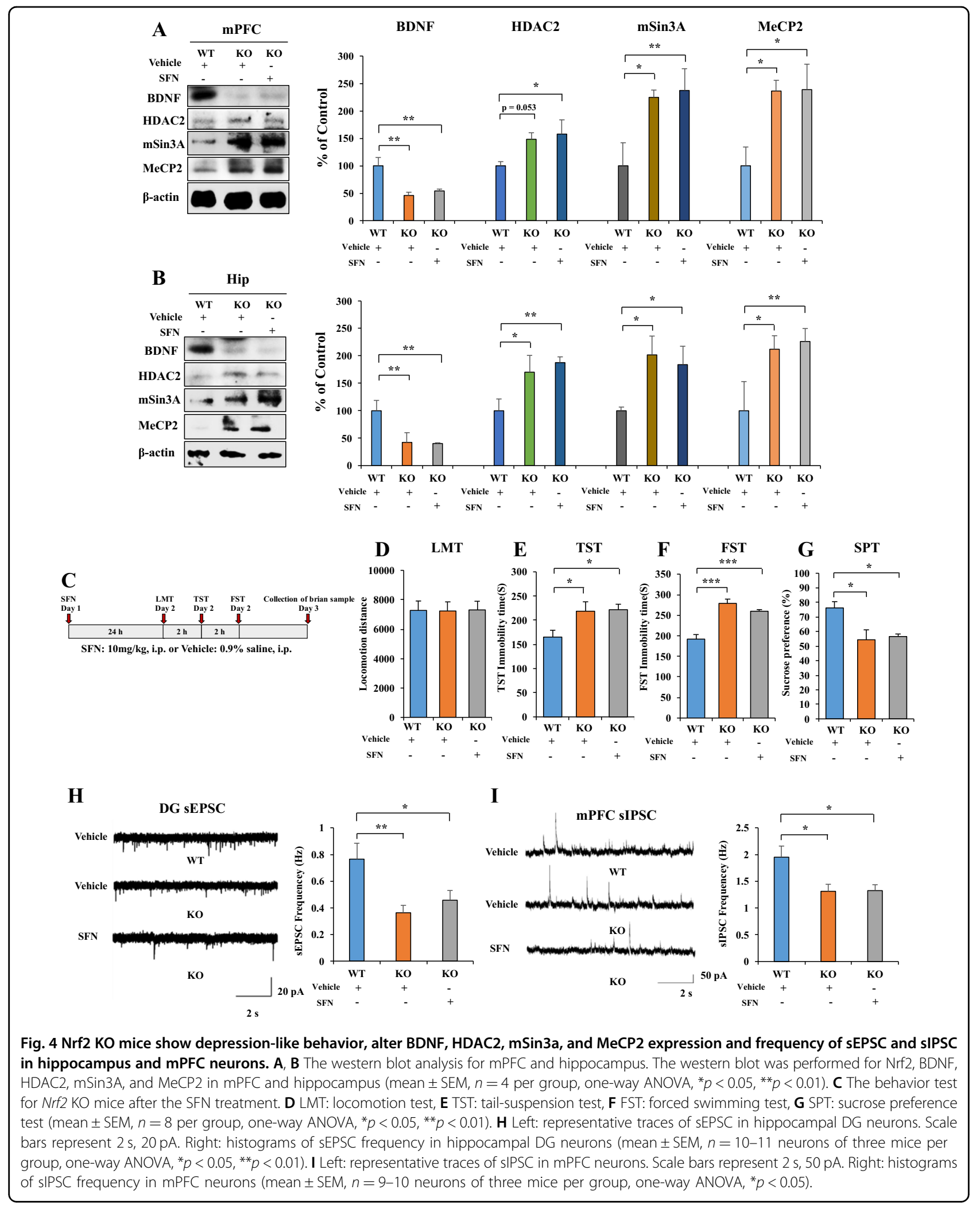




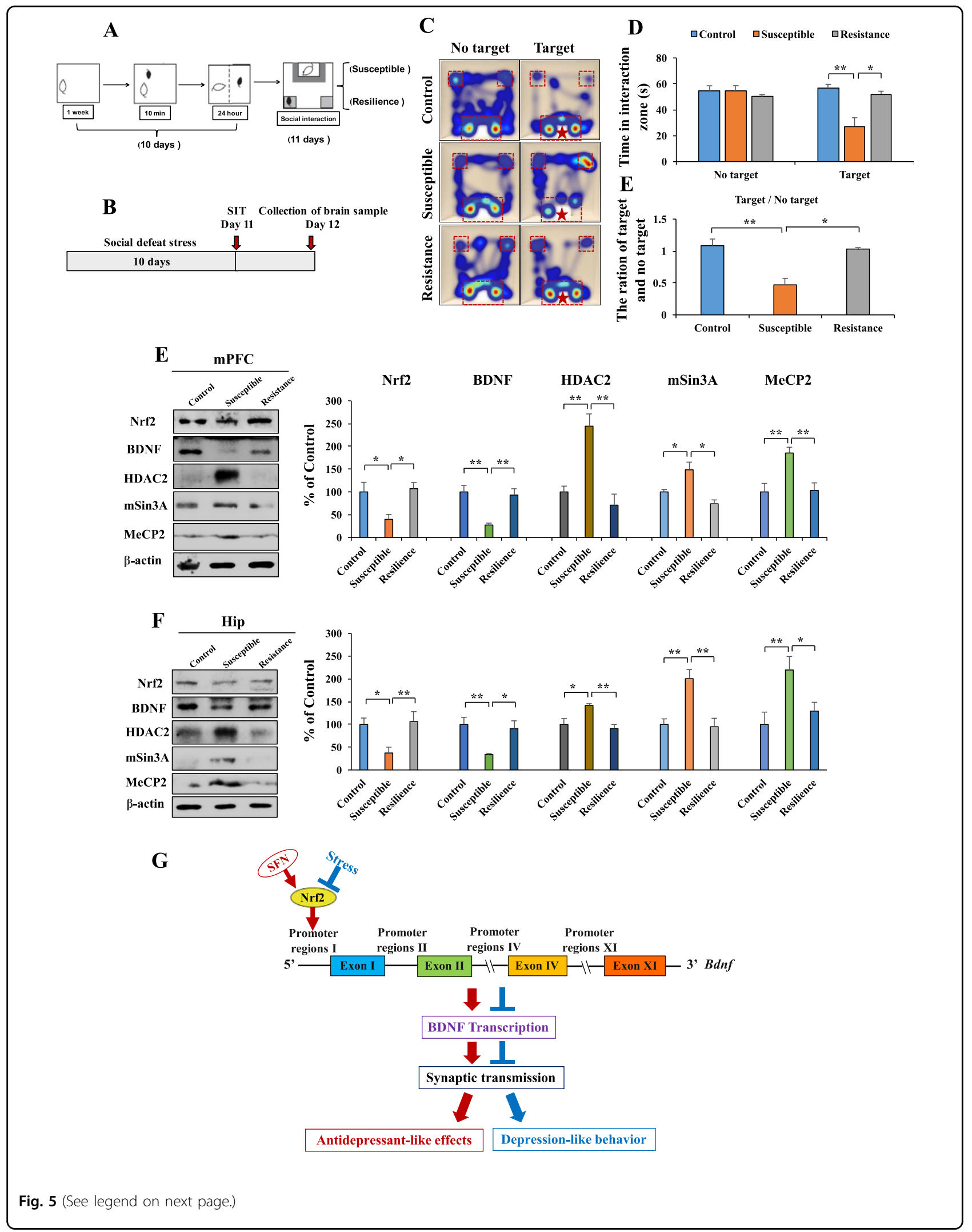


(see figure on previous page)

Fig. 5 Resistance elevates Nrf2, BDNF expression and inhibits HDAC2, mSin3a, and MeCP2 expression. A, B Schematic of social defeat stress model and the schedule of treatment. C Thermal imaging of mice trajectories. D The social interaction test for no target and target time (mean \pm SEM, $n=4-6$ per group, one-way ANOVA, ${ }^{*} p<0.05,{ }^{* *} p<0.01$ ). E The ration for target and no target time (mean \pm SEM, $n=4-6$ per group, one-way ANOVA, $\left.{ }^{*} p<0.05,{ }^{* *} p<0.01\right)$. E, F The western blot analysis for mPFC and hippocampus. The western blot was performed for Nrf2, BDNF, HDAC2, $\mathrm{mSin} 3 \mathrm{~A}$, and MeCP2 in mPFC and hippocampus (mean $\pm \mathrm{SEM}, n=4$ per group, one-way ANOVA, ${ }^{*} p<0.05,{ }^{* *} p<0.01$ ). G The working model of Nrf2induced BDNF transcription in the model of depression. Stress inhibits Nrf2 expression, which leads to inhibit BDNF transcriptional and abnormal synaptic transmission, causing depression-like behaviors in mice. SFN induces BDNF transcription by activating Nrf2 and could correct the abnormal synaptic transmission, resulting in antidepressant-like effects.

\section{Discussion}

The major findings of this study are as follows. First, the activation of Nrf2 by SFN or Nrf2 gene transfection increased the expression of $B d n f$ mRNA and protein while decreasing the expression of its transcriptional repressors (HDAC2, mSin3a, and MeCP2) in Neuro-2a cells. These effects were blocked by siRNA-Nrf2, suggesting a role for Nrf2. Second, using the luciferase and ChIP assays, we found that Nrf2 binds to the $B d n f$ exon I promoter, indicating that Nrf2 is a transcription factor for $B d n f$. Third, we found decreased expression of Nrf2 and BDNF protein levels in the MPFC and hippocampus from LPStreated mice, whereas the protein expression of BDNF transcriptional repressors (HDAC2, mSin3a, and MeCP2) was increased in LPS-treated mice. Fourth, SFN showed antidepressant-like effects by normalizing the decreased levels of Nrf2 and BDNF protein; increased levels of HDAC2, mSin3a, and MeCP2 protein; and abnormal synaptic transmission observed in LPS-treated mice. Fifth, SFN did not show antidepressant-like effects nor ameliorate the altered expression of BDNF, HDAC2, $\mathrm{mSin} 3 \mathrm{~A}$, and McCP2; and abnormal synaptic transmission observed in the mPFC and hippocampus of Nrf2 KO mice. Finally, we found that decreased expression of Nrf2 and BDNF as well as increased expression of HDAC2, $\mathrm{mSin} 3 \mathrm{~A}$, and MeCP2 in the mPFC and hippocampus might confer stress susceptibility in mice after CSDS. The downregulation of BDNF caused by reduced Nrf2 activity may play a key role in depression-like phenotypes in rodents.

It is reported that Nrf2 binds to the antioxidant responsive element, which is located in the promoter region of genes encoding many detoxifying or antioxidant enzymes and related stress-responsive proteins ${ }^{2-4,6}$. We previously reported that pretreatment with SFN or dietary intake of $0.1 \%$ glucoraphanin (a precursor for SFN) significantly attenuated depression-like phenotypes and altered BDNF expression in the MPFC and hippocampus of mice with depression-like phenotypes ${ }^{10,11}$. Using the luciferase and ChIP assays, we confirmed that Nrf2 binds to the $B d n f$ exon I promoter at two binding sites. Using immunofluorescence, we observed the SFN-induced redistribution of Nrf2 and MeCP2 for the nucleus. We found that more Nrf2 within the nucleus and the more diffused nuclear staining pattern of MeCP2 in vehicletreated cells became more punctate upon SFN treatment. These data suggest that Nrf2 functions as a transcription factor for $B d n f$.

Nrf2 can interact with its principal negative regulator, the E3 ligase adapter Kelch-like ECH-associated protein $(\text { Keap } 1)^{2-7}$. We reported decreased expression of Nrf2 and Keap1 in the parietal cortex from MDD patients compared with that from the control group, which indicates that decreased Keap1-Nrf2 signaling may play a crucial role in the development of depression ${ }^{14}$. We also reported alterations in the expression of BDNF, BDNF pro-peptide, and their precursor (proBDNF) in the parietal cortex of patients with major psychiatric disorders, including $\mathrm{MDD}^{45}$. Thus, abnormalities in the processing of BDNF and BDNF pro-peptide from proBDNF in the parietal cortex may contribute to the pathogenesis of major psychiatric disorders. Furthermore, Bouvier et al ${ }^{46}$ showed that the reduced levels of BDNF in mice with depressionlike behavior could prevent Nrf2 translocation and the activation of detoxifying/antioxidant enzymes, which ultimately sustained oxidative stress. Furthermore, it is suggested that BDNF plays a key role as an inducer of neuronal antioxidant responses by BDNF-induced Nrf2 nuclear translocation ${ }^{47}$. A recent study demonstrated the role of BDNF-TrkB signaling in the antidepressant-like effects of $(R)$-ketamine in $\mathrm{Nrf2} \mathrm{KO}$ mice $^{48}$. Therefore, it is likely that the communication between Keap1-Nrf2 signaling and BDNF-TrkB signaling in the brain might play a crucial role in depression (Fig. 5G).

Rodents with depression-like phenotypes show impaired glutamatergic signaling in the hippocampus and GABAergic transmission in the $\mathrm{mPFC}^{40,49}$. LPS administration impaired the sEPSC frequency of hippocampal neurons and SIPSC frequency of mPFC neurons. Also, SFN reversed impairments in the glutamatergic transmission of hippocampal neurons and GABAergic transmission in mPFC neurons among LPS-treated mice. We found that sEPSC frequency of hippocampal neurons and sIPSC frequency of mPFC neurons of Nrf2 $\mathrm{KO}$ mice are abnormal, and that SFN could not reverse impairments in the glutamatergic transmission of hippocampal neurons and GABAergic transmission in mPFC neurons of Nrf2 $\mathrm{KO}$ mice. Considering the role of $\mathrm{Nrf} 2$ as the transcription 
factor for $B d n f$, we propose that the activation of Nrf2 may ameliorate LPS-induced impairments in the glutamatergic transmission of hippocampal neurons and GABAergic transmission of mPFC neurons through BDNF activation. Previous studies showed that the deletion of $B d n f$ could decrease the frequency of sEPSCs in the hippocampus ${ }^{50}$, and $B d n f$ promotor IV mutant mice exhibited significant deficits in GABAergic transmission in the $\mathrm{mPFC}^{51}$. Collectively, LPS-induced suppression of Nrf2 expression and subsequent inhibition of $B d n f$ transcription may contribute to depression-like phenotypes and abnormal synaptic transmission in the mice with depression-like phenotype. However, further studies are needed.

Multiple lines of evidence support that resilience is implicated in the development of several psychiatric disorders $^{52,53}$. However, the detailed molecular mechanisms underlying resilience against MDD remain unknown. We previously showed that alterations in the levels of BDNF, BDNF pro-peptide, and pro-BDNF in the $\mathrm{mPFC}$ and hippocampus confer stress resilience in the rat $\mathrm{LH}$ mode $\mathrm{l}^{30}$. Using the same samples, we reported that the protein levels of Keap1 and Nrf2 in the mPFC and DG of hippocampi from LH (susceptible) rats were lower than those in the control and non-LH (resilience) rats. Therefore, Keap1-Nrf2 signaling may play a role in stress resilience ${ }^{14}$. It is also reported that activation of Nrf2 translocation decreased vulnerability to depres$\operatorname{sion}^{46}$. A recent study demonstrated that decreased Keap1-Nrf2 signaling in the mPFC, hippocampus, and muscle might increase susceptibility to anhedonia after spared nerve injury (SNI) surgery. Furthermore, SFN exerts beneficial effects in SNI rats by attenuating decreased Keap1-Nrf2 signaling ${ }^{54}$. Decreased Nrf2 expression might decrease BDNF expression in the $\mathrm{mPFC}$ and hippocampus, resulting in a depression-like phenotype. We found that CSDS-resilient mice showed increased levels of $\mathrm{Nrf} 2$ and decreased expression of HDAC2, mSin3a, and MeCP2 in the MPFC and hippocampus compared with CSDS-susceptible mice. Moreover, based on our in vitro studies, Nrf2 binding to the $B d n f$ exon I promoter results in $B d n f$ transcription. Therefore, the inhibition of Nrf2-induced Bdnf transcription may contribute to CSDS-induced depressionlike behaviors, and activation of Nrf2-induced $B d n f$ transcription may confer stress resilience (Fig. 5G).

In conclusion, $\mathrm{Nrf} 2$ regulates the transcription of $B d n f$ by binding to its exon I promoter. Furthermore, the inhibition of Nrf2-induced $B d n f$ transcription may play a role in the pathophysiology of depression. Also, the activation of Nrf2-induced $B d n f$ transcription promoted antidepressant-like effects. Finally, alterations in the crosstalk between Nrf2 and BDNF may contribute to resilience versus susceptibility after stress.

\section{Acknowledgements}

This work is supported by the National Natural Science Foundation of China (81973341 to Q.Q., 31900825 to S.L., 81822016 and 81771382 to Z.Z., 81630104 to J.C.), the Fundamental Research Funds for the Central Universities (11620425 to J.Z.), the Science and Technology Program of Guangzhou (202002030010 to Q.Q.), Huang Zhendong Research Fund for Traditional Chinese Medicine of Jinan University (201911 to J.C.), grant-in-aid for Scientific Research (B) of Japan Society for the Promotion of Science (17H04243 to K.H.), and Japan Agency for Medical Research and Development (JP20dm0107119 to K.H.). The authors are thankful to Dr. Xuesong Yang and Dr. Guang Wang at Key Laboratory for Regenerative Medicine of the Ministry of Education, Division of Histology and Embryology, Medical College, Jinan University, for providing the Nrf2 KO mice.

\section{Author details}

${ }^{1}$ Formula-Pattern Research Center, School of Traditional Chinese Medicine, Jinan University, 510632 Guangzhou, China. ${ }^{2}$ Department of Physiology, School of Medicine, Jinan University, 510632 Guangzhou, China. ${ }^{3}$ MOE Key Laboratory of Tumor Molecular Biology; Clinical Translational Center for Targeted Drug, Department of Pharmacology, School of Medicine, Jinan University, 510632 Guangzhou, China. ${ }^{4}$ Guangzhou Overseas Chinese Hospital of Jinan University, 510632 Guangzhou, China. ${ }^{5}$ Department of Neurology, Renmin Hospital of Wuhan University, 430060 Wuhan, China. ${ }^{6}$ Division of Clinical Neuroscience, Chiba University Center for Forensic Mental Health, Chiba 260-8670, Japan

\section{Author contributions}

J.Z., Q.Q., and K.H. conceived the project, designed the experiments, analyzed the data, and wrote the manuscript. W.Y. designed and performed most of the experiments and analyzed the data. S.L. performed the electrophysiology, analyzed the data, and wrote the manuscript. J.S. performed the western blot for the samples of in vitro study. Q.C. performed the Western blot for the samples of in vivo study. Y.C. performed the social defeat stress model. J.C. and Z.Z. assisted with data analysis and interpretation, and critically read the manuscript.

\section{Conflict of interest}

The authors declare no competing interests.

\section{Publisher's note}

Springer Nature remains neutral with regard to jurisdictional claims in published maps and institutional affiliations.

Supplementary information The online version contains supplementary material available at https://doi.org/10.1038/s41398-021-01261-6.

Received: 6 November 2020 Accepted: 1 February 2021

Published online: 24 February 2021

\section{References}

1. World Health Organization (WHO). Depression. https://www.who.int/newsroom/fact-sheets/detail/depression (2020)

2. Suzuki, T. \& Yamamoto, M. Molecular basis of the Keap1-Nrf2 system. Free Radic. Biol. Med. 88, 93-100 (2015).

3. Suzuki, T., Motohashi, H. \& Yamamoto, M. Toward clinical application of the Keap1-Nrf2 pathway. Trends Pharmacol. Sci. 34, 340-346 (2013).

4. Ma, Q. Role of nrf2 in oxidative stress and toxicity. Annu. Rev. Pharmacol. Toxicol. 53, 401-426 (2013)

5. Kometsi, L., Govender, K., Mofo Mato, E. P., Hurchund, R. \& Owira, P. M. O. By reducing oxidative stress, naringenin mitigates hyperglycaemia-induced upregulation of hepatic nuclear factor erythroid 2-related factor 2 protein. J. Pharm. Pharmacol. 72, 1394-1404 (2020).

6. Kobayashi, E., Suzuki, T. \& Yamamoto, M. Roles Nrf2 plays in myeloid cells and related disorders. Oxid. Med. Cell. Longev. 2013, 529219 (2013).

7. Yamamoto, M., Kensler, T. W. \& Motohashi, H. The KEAP1-NRF2 System: a thiolbased sensor-effector apparatus for maintaining redox homeostasis. Physiol. Rev. 98, 1169-1203 (2018).

8. Hashimoto, K. Essential role of Keap1-Nrf2 signaling in mood disorders: overview and future perspective. Front. Pharmacol. 9, 1182 (2018). 
9. Mendez-David, I. et al. Nrf2-signaling and BDNF: a new target for the antidepressant-like activity of chronic fluoxetine treatment in a mouse model of anxiety/depression. Neurosci. Lett. 597, 121-126 (2015).

10. Zhang, J. C. et al. Prophylactic effects of sulforaphane on depression-like behavior and dendritic changes in mice after inflammation. J. Nutr. Biochem. 39, 134-144 (2017).

11. Yao, W. et al. Role of Keap1-Nrf2 signaling in depression and dietary intake of glucoraphanin confers stress resilience in mice. Sci. Rep. 6, 30659 (2016).

12. Yao, W. et al. Antidepressant effects of TBE-31 and MCE-1, the novel Nrf2 activators, in an inflammation model of depression. Eur. J. Pharmacol. 793 21-27 (2016)

13. Nakayama, T. et al. Seasonal changes in NRF2 antioxidant pathway regulates winter depression-like behavior. Proc. Natl Acad. Sci. USA 117, 9594-9603 (2020).

14. Zhang, J. C. et al. Keap1-Nrf2 signaling pathway confers resilience versus susceptibility to inescapable electric stress. Eur. Arch. Psychiatry Clin. Neurosci. 268, 865-870 (2018)

15. Yang, C., Shirayama, Y., Zhang, J. C., Ren, Q. \& Hashimoto, K. Regional differences in brain-derived neurotrophic factor levels and dendritic spine density confer resilience to inescapable stress. Int. J. Neuropsychopharmacol. 18, pyu121 (2015).

16. Nestler, E. J. et al. Neurobiology of depression. Neuron 34, 13-25 (2002).

17. Lindholm, J. S. \& Castrén, E. Mice with altered BDNF signaling as models for mood disorders and antidepressant effects. Front. Behav. Neurosci. 8, 143 (2014).

18. Hashimoto, K., Shimizu, E. \& lyo, M. Critical role of brain-derived neurotrophic factor in mood disorders. Brain Res. Rev. 45, 104-114 (2004).

19. Castrén, E. Neurotrophins and psychiatric disorders. Handb. Exp. Pharmacol. 220, 461-479 (2014)

20. Bjorkholm, C. \& Monteggia, L. M. BDNF - a key transducer of antidepressant effects. Neuropharmacology 102, 72-79 (2016).

21. Hashimoto, K. Brain-derived neurotrophic factor as a biomarker for mood disorders: an historical overview and future directions. Psychiatry Clin. Neurosci. 64, 341-357 (2010).

22. Hashimoto, K. Rapid-acting antidepressant ketamine, its metabolites and other candidates: a historical overview and future perspective. Psychiatry Clin. Neurosci. 73, 613-627 (2019).

23. Hashimoto, K. Molecular mechanisms of the rapid-acting and long-lasting antidepressant actions of (R)-ketamine. Biochem. Pharmacol. 177, 113935 (2020).

24. Zhang, J. C., Yao, W. \& Hashimoto, K. Brain-derived neurotrophic factor (BDNF)TrkB signaling in inflammation-related depression and potential therapeutic targets. Curr. Neuropharmacol. 14, 721-731 (2016).

25. Yang, C. et al. R-ketamine: a rapid-onset and sustained antidepressant without psychotomimetic side effects. Transl. Psychiatry 5, e632 (2015).

26. Wei, I. H. et al. Acute amino acid D-serine administration, similar to ketamine, produces antidepressant-like effects through identical mechanisms. J. Agric. Food Chem. 65, 10792-10803 (2017).

27. Zhang, J. C. et al. Comparison of ketamine, 7,8-dihydroxyflavone, and ANA-12 antidepressant effects in the social defeat stress model of depression. Psychopharmacology 232, 4325-4335 (2015).

28. Zhang, J. C. et al. Antidepressant effects of TrkB ligands on depression-like behavior and dendritic changes in mice after inflammation. Int. J. Neuropsychopharmacol. 18, pyu077 (2014).

29. Shirayama, Y. et al. Alterations in brain-derived neurotrophic factor (BDNF) and its precursor proBDNF in the brain regions of a learned helplessness rat model and the antidepressant effects of a TrkB agonist and antagonist. Eur. Neuropsychopharmacol. 25, 2449-2458 (2015).

30. Yang, B. et al. Regional differences in the expression of brain-derived neurotrophic factor (BDNF) pro-peptide, proBDNF and preproBDNF in the brain confer stress resilience. Eur. Arch. Psychiatry Clin. Neurosci. 266 765-769 (2016)

31. Taliaz, D. et al. Resilience to chronic stress is mediated by hippocampal brainderived neurotrophic factor. J. Neurosci. 31, 4475-4483 (2011).
32. Koponen, E. et al. Transgenic mice overexpressing the full-length neurotrophin receptor trkB exhibit increased activation of the TrkB-PLCgamma pathway, reduced anxiety, and facilitated learning. Mol. Cell. Neurosci. 26, 166-181 (2004).

33. Koponen, E. et al. Enhanced BDNF signaling is associated with an antidepressant-like behavioral response and changes in brain monoamines. Cell. Mol. Neurobiol. 25, 973-980 (2005).

34. Koponen, E., Lakso, M. \& Castren, E. Overexpression of the full-length neurotrophin receptor trkB regulates the expression of plasticity-related genes in mouse brain. Brain Res.ÿMol. Brain Res. 130, 81-94 (2004).

35. Berton, O. et al. Essential role of BDNF in the mesolimbic dopamine pathway in social defeat stress. Science 311, 864-868 (2006).

36. Golden, S. A., Covington, H. E. 3rd, Berton, O. \& Russo, S. J. A standardized protocol for repeated social defeat stress in mice. Nat. Protoc. 6, 1183-1191 (2011).

37. Tsankova, N. M. et al. Sustained hippocampal chromatin regulation in a mouse model of depression and antidepressant action. Nat. Neurosci. 9, 519-525 (2006).

38. Zhao, T. et al. Effects of chronic social defeat stress on behavior and choline acetyltransferase, 78-kDa glucose-regulated protein, and CCAAT/enhancerbinding protein (C/EBP) homologous protein in adult mice. Psychopharmacology (Berl.) 228, 217-230 (2013).

39. Chorley, B. N. et al. Identification of novel NRF2-regulated genes by ChIP-Seq influence on retinoid X receptor alpha. Nucleic Acids Res. 40, 7416-7429 (2012).

40. Duman, R. S. Ketamine and rapid-actin $g$ antidepressants: a new era in the battle against depression and suicide. F1000Res. 7, 659 (2018).

41. Kang, H. J. et al. Decreased expression of synapse-related genes and loss of synapses in major depressive disorder. Nat. Med. 18, 1413-1417 (2012).

42. Li, $\mathrm{N}$. et al. Glutamate $\mathrm{N}$-methyl-D-aspartate receptor antagonists rapidly reverse behavioral and synaptic deficits caused by chronic stress exposure. Biol. Psychiatry 69, 754-761 (2011).

43. Leal, G., Comprido, D. \& Duarte, C. B. BDNF-induced local protein synthesis and synaptic plasticity. Neuropharmacology 76, 639-656 (2014).

44. Ghosal, S., Hare, B. \& Duman, R. S. Prefrontal cortex GABAergic deficits and circuit dysfunction in the pathophysiology and treatment of chronic stress and depression. Curr. Opin. Behav. Sci. 14, 1-8 (2017).

45. Yang, B., Ren, Q., Zhang, J. C., Chen, Q. X. \& Hashimoto, K. Altered expression of BDNF, BDNF pro-peptide and their precursor proBDNF in brain and liver tissues from psychiatric disorders: rethinking the brain-liver axis. Transl. Psychiatry 7, e1128 (2017).

46. Bouvier, E. et al. Nrf2-dependent persistent oxidative stress results in stressinduced vulnerability to depression. Mol. Psychiatry 22, 1701-1713 (2017).

47. Qu, Y. et al. Rapid-acting and long-lasting antidepressant-like action of (R)ketamine in Nrf2 knock-out mice: a role of TrkB signaling. Eur. Arch. Psychiatry Clin. Neurosci. https://doi.org/10.1007/s00406-020-01208-w (2020).

48. Bruna, B. et al. The signaling pathways underlying BDNF-induced Nrf2 hippocampal nuclear translocation involve ROS, RyR-Mediated $\mathrm{Ca}^{2+}$ signals, ERK and PI3K. Biochem. Biophys. Res. Commun. 505, 201-207 (2018).

49. Yang, Y., Ju, W., Zhang, H. \& Sun, L. Effect of ketamine on LTP and NMDAR EPSC in hippocampus of the chronic social defeat stress mice model of depression. Front. Behav. Neurosci. 12, 229 (2018).

50. Edelmann, E. et al. Theta burst firing recruits BDNF release and signaling in postsynaptic CA1 neurons in spike-timing-dependent LTP. Neuron $\mathbf{8 6}$, 1041-1054 (2015)

51. Sakata, K et al. Critical role of promoter IV-driven BDNF transcription in GABAergic transmission and synaptic plasticity in the prefrontal cortex. Proc. Natl Acad. Sci. USA 106, 5942-5947 (2009).

52. Feder, A., Nestler, E. J. \& Charney, D. S. Psychobiology and molecular genetics of resilience. Nat. Rev. Neurosci. 10, 446-457 (2009).

53. Russo, S. J., Murrough, J. W., Han, M. H., Charney, D. S. \& Nestler, E. J. Neurobiology of resilience. Nat. Neurosci. 15, 1475-1484 (2012).

54. Li, S. et al. Role of Keap1-Nrf2 signaling in anhedonia symptoms in a rat model of chronic neuropathic pain: improvement with sulforaphane. Front. Pharmacol. 9, 887 (2018). 